\title{
Study On the Radical Basis Function Neural Network Based On Niche Genetic Algorithms
}

\author{
Zhaohu Deng ${ }^{1,2, a}$ \\ ${ }^{1}$ School of Mechanical and Automotive Engineering South China University of Technology \\ Guangzhou, China \\ ${ }^{2}$ Mechanical and Electrical Engineering Department Guangdong Polytechnic College Guangzhou, \\ China \\ a360596374@qq.com
}

Keywords: RBF, niche genetic algorithms, optimization, global search

Abstract. When constructing a radial basis function (RBF) neural network with clustering method , the prediction of the RBF neural network is usually influenced by the distribution of training samples.The quality of RBF neural network are difficult to catch the best.In this paper, it presents to through changing the traditional method with the niche GA to solve the matters above. Through tests, it showed that the quality of new RBF neural network built by niche genetic algorithms(NGA) performed better than the traditional one.

\section{Introduction}

RBF neural network is a kind of three-layer feed forward neural network[1].Because it can map the function excellently, the RBF neural network has been extensively applied in many engineering fields. In RBF neural network, the centers and width of radial basis function and the connection weights between hidden layer and output layer are important. The process of constructing RBF neural network is to determine the two parameters actually. After that it would establish the weights between the hidden layer and the output layer. This process is mainly determined by Least Mean Squares (LMS) algorithm [2].

Though the RBF neural network can map any nonlinear function to an arbitrary degree of accuracy [3]. The process of constructing a proper RBF neural network were difficult. If the number of basis functions is fewer, the built RBF neural network can' t fit the training data enough. If the number of basis functions is excessive, these basis functions would be influenced by the noise of the training samples. So constructing a RBF neural network was an optimization process to get the best number of the radical base function. In this paper it achieved this purpose depending on niche GA.

\section{Optimization of RBF Neural Network}

Because the GA has the characteristics of global optimization, it is helpful to avoid the center of RBF being trapped in local optimum. In this paper it defined the center of RBF as optimization variable, and the performance function of RBF as objective function. First it will determine the number of hidden layout neural and the center of RBF with GA. Then it will determine the weights of output layer with supervised learning rule.

Parameters definition of GA. GA is a kind of adaptive global optimization algorithm, which imitates the processes of evolution and heredity. It is especially suitable for the optimization of large scale, highly nonlinear discontinuous multi-peak function, and the optimization of the objective function without analytic expression. The basic parameters of GA include encoding method, fitness evaluation and genetic operator.For different problems, the definition of parameters will be different.In this paper it took parameters definition as follow.

Encoding method. The purpose of encoding is to convert the solution space of problems into the search space in GA. In this paper it adopted binary encoding. And the encoding length would be limited by the training samples. For resolving the matters it present to encode the serial number of 
samples rather than the samples. Thus this method will be applied for more different optimization case.

Fitness evaluation. The fitness value of every individual determines its probability to inherit to the next generation. So the fitness of individual was defined as Eq. 1 and Eq. 2.

$$
\begin{aligned}
& F=\frac{1}{2} \sum_{i=1}^{N} e_{j}^{2} \\
& e_{j}=d_{j}-\sum_{i=1}^{m} w_{i} \exp \left(-\frac{\left\|x_{i}-t_{i}\right\|}{2 \sigma_{i}^{2}}\right)
\end{aligned}
$$
output.

$\mathrm{N}$ is the number of training samples, $\mathrm{m}$ is the number of hidden cells, $\mathrm{d}_{\mathrm{j}}$ is the value of desired

Genetic operator. There are three operators in GA, it involves selection operator, mutation operator and crossover operator.Selection operator is benefit to find the local optimum. Mutation operator is benefit to enlarge the search range. Crossover operator is used to produce a new individual.In this paper it used proportional selection operator, uniform mutation operator and single random crossover operator .

Improvement of GA. Because GA tends to trapped into the premature,it resolved the problem with niche technology in this paper. In the early stage of optimization, it would compare the fitness of different individual. If the individual had poor fitness and its hamming distance was just lower than the set value, then it would have more chance to be eliminated. Thus it could ensure that there was only one quality individual existing within the hamming distance. It was useful to make the RBF center be scattered in the global search scope.

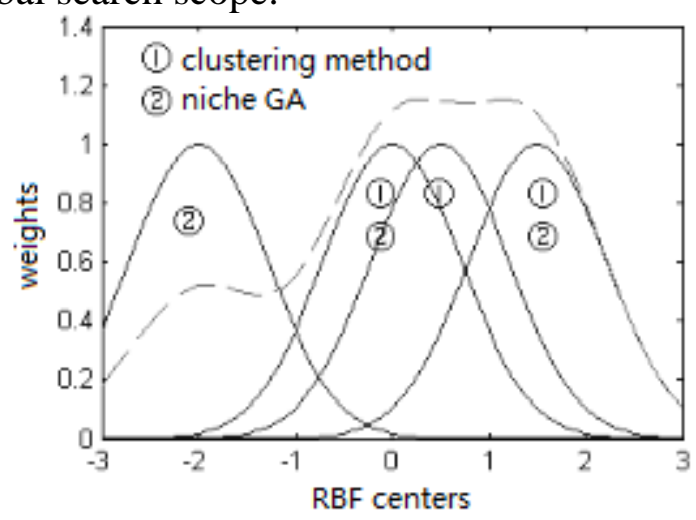

Fig. 1 The scheme of RBF center choice with different methods

Fig. 1 shows the distribution difference of RBF center with different method. It illustrates that the niche GA could raise the global response ability of RBF neural network. Fig. 2 shows the procedure of constructing RBF neural network with niche GA. 


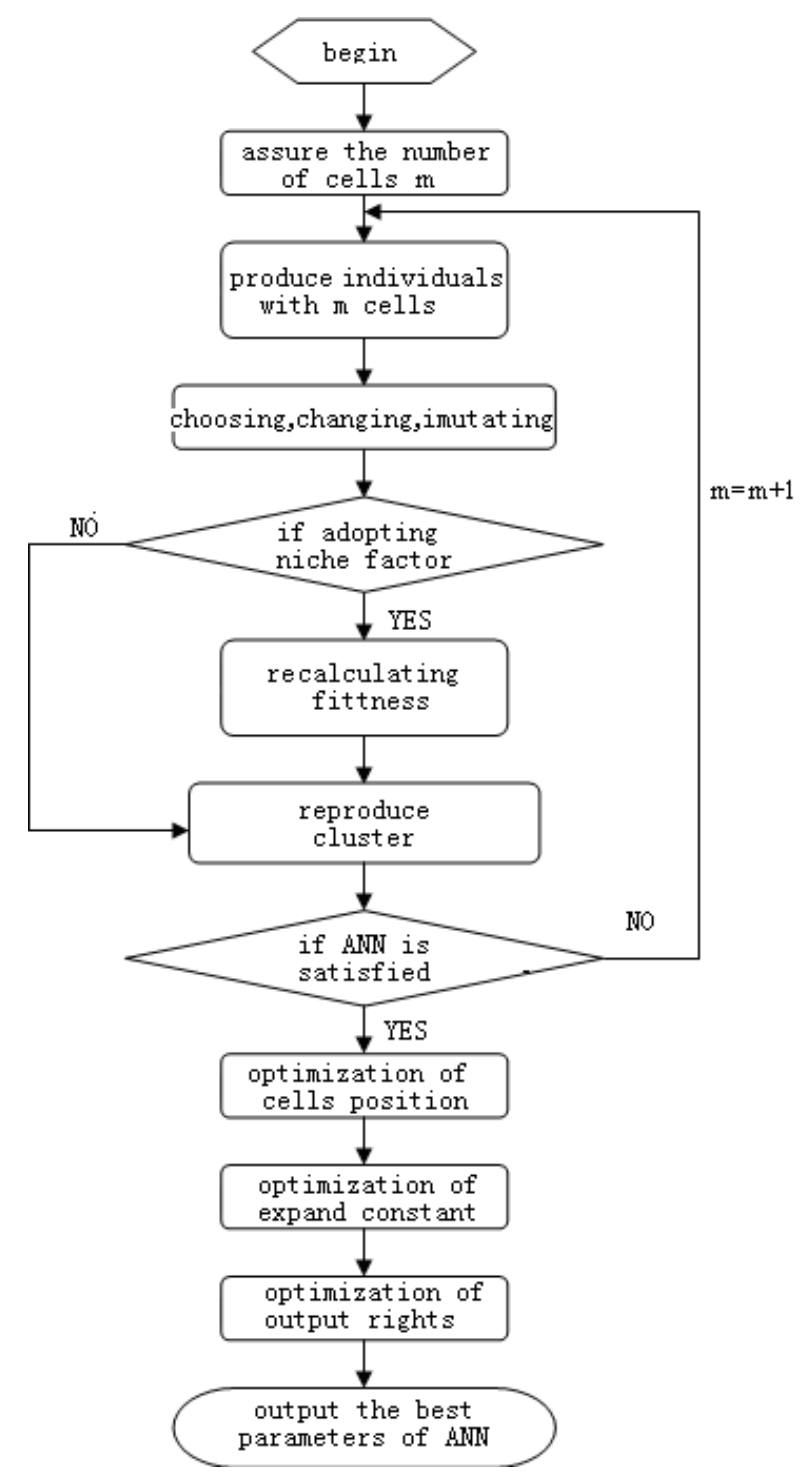

Fig.2 The procedure chart of building RBF neural network

\section{Experiments}

For verifying the availability of RBF neural network based on niche GA, it took 100 sets of data as the training samples. The important parameters were defined as: original scale $m=30$, number of iteration $\mathrm{T}=500$, selection probability $\mathrm{ps}=0.5$, mutation probability $\mathrm{pm}=0.03$, crossover probability $\mathrm{pc}=0.5$, niche factor $\mathrm{L}=0.5$. After 410 times iteration, the $\mathrm{RBF}$ neural network got the optimum performance.Fig. 3 compared the mapping ability of RBF neural network with different method. It showed that the RBF neural network built based on niche GA will predict more accurately than the one constructed with clustering algorithm.

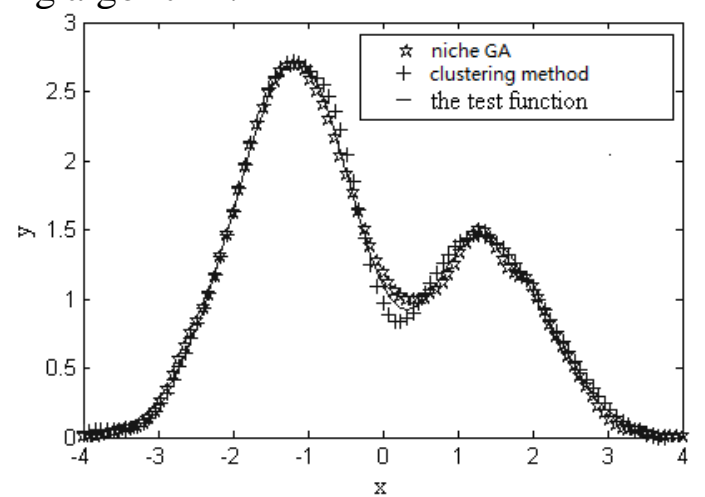

Fig.3 The prediction of RBF neural network with different methods 


\section{Summary}

In this paper a new method of building RBF neural network was presented. It benefit the excellence of GA in global optimization It not only got the optimal RBF centers, but also made them distribute reasonably. In order to test the quality of the proposed method, it compared the proposed method with the traditional clustering method. The result showed that the RBF neural network constructed with niche GA could map the function more accurately than the one constructed with traditional method.

\section{Acknowledgements}

This work was financially supported by the Project of Research and Production Combination Financed by Guangzhou. Project number: 201392-00084.

\section{References}

[1]. Sultan Noman Qasem, Siti Mariyam Shamsuddin. Hybrid Learning Enhancement of RBF Network Based on Particle Swarm Optimization. 6th International Symposium on Neural Networks, ISNN 2009, Proceedings, : 19-29.

[2]. Liu, Y., Zheng, Q., Shi, Z., Chen, J.: Training Radial Basis Function Networks with Particle Swarms. In: Yin, F.-L., Wang, J., Guo, C. (eds.) ISNN 2004. LNCS, vol. 3173,: 317 - 322. Springer, Heidelberg (2004).

[3]. Min Han, Wei Guo, Yunfeng Mu. A Modified RBF Neural Network in Pattern Recognition.Proceedings of International Joint Conference on Neural Networks, Orlando,Florida, USA, August 12-17, 2007. 